\title{
Virtual based Data Dissemination Schemes for Mobile Sink in WSN
}

\author{
Rinki Thakur \\ Student MTech.CSE Dept., \\ Sri Sai University Palampur \\ H.P.(India)
}

\author{
Yamini Sood \\ Astt. Prof. CSE Dept., \\ Sri Sai University Palampur \\ H.P.(India)
}

\begin{abstract}
In wireless sensor networks, emergence of sink mobility has been considered as a good strategy to maintain the nodes energy dissipation. According to previous approaches the Dynamic Routes Adjustment (VGDRA) plan that acquires slightest correspondence cost while keeping up almost ideal courses to the most recent area of the portable sink. The proposed plan parcels the sensor field into a virtual matrix and develops a virtual spine structure included the cell header hubs. A versatile sink while moving around the sensor field continues changing its area and associates with the nearest marginal cell-header for information accumulation. Utilizing a set of correspondence guidelines, limited number of the cellheaders participates in the courses recreation prepares along these lines diminishing the general correspondence cost. The proposed approach is to minimize energy utilization and maximize network lifetime by implementing optimization technique Biogeography Based Optimization (BBO) as selection algorithm for route adjustment. The simulation results represented improved network lifetime of existed VGDRA comparison with VGDRA-BBO using ns2 as a simulation tool.
\end{abstract}

\section{General Terms}

Virtual Grid, Route Reconstruction, Data Dissemination, Dynamic network topology

\section{Keywords}

Wireless Sensor Network, Life time, Throughput, Mobile sink

\section{INTRODUCTION}

Wireless Sensor Network (WSN) - a self-composed system of modest registering and specialized gadgets (sensor nodes) has been generally utilized as a part of a few un-went to and perilous situations. In a commonplace organization of WSN, hubs are battery worked where they agreeably screen and report some wonder of enthusiasm to a focal hub called sink or base-station for further handling and examination. Conventional static hubs arrangement where hubs display nto- 1 correspondence in reporting their watched information to a solitary static sink, offers ascend to vitality opening wonder in the region of sink. Sink versatility presented in [1] not just adjusts the hub's vitality scattering can segregated system fragments in dangerous zones [2].

Likewise, a couple application circumstances regularly require sink mobility in the sensor field [3] e.g., in a flood organization structure, a rescuer outfitted with a PDA can move around the unsafe circumstance to scan for any survivor. So additionally, in a battle zone environment, a chairman can procure constant information about any intrusion of enemies, size of ambush, suspicious activities thus on through field sensors while moving. In an Intelligent Transport System (ITS), sensor centroid sent at various purposes of interest - crossing points, auto parks, zones vulnerable to falling rocks, can give early notification to drivers (adaptable sink) well before their physical technique. Mishandling the sink's flexibility draws out the framework lifetime thusly moderating imperativeness hole issue; regardless, it brings new challenges for the data diffusing procedure.

Exploiting the sink's mobility helps to prolong the network lifetime thereby alleviating energy-hole problem; however, it brings new challenges for the data dissemination process. Unlike static sink scenarios, the network topology becomes dynamic as the sink keeps on changing its location [4]. To cope with the dynamic network topology, nodes need to keep track of the latest location of the mobile sink for efficient data delivery. Some data dissemination protocols e.g., Directed Diffusion [5], where the periodic flooding takes place leads to more collision and retransmission. Taking into consideration the scarce energy resource of nodes, frequent propagation of sink's mobility updates should be avoided as it greatly undermines the energy conservation goal. In this regard, to enable sensor nodes to maintain fresh routes towards the mobile sink while incurring minimal communication cost, overlaying based virtual infrastructure over the physical network is considered as an efficient approach [6]. In the virtual infrastructure based data dissemination schemes, only a set of designated nodes scattered in the sensor field are responsible to keep track of sink's location. Such designated nodes gather the observed data from the nodes in their vicinity during the absence of the sink and then proactively or reactively report data to the mobile sink.

In this paper, a novel scheme called Virtual Grid based Dynamic Routes Adjustment (VGDRA) is proposed for periodic data collection from WSN [15]. It aims to optimize the trade-off between nodes energy consumption and data delivery performance using a single mobile sink in WSN. The proposed scheme enables sensor nodes to maintain nearly optimal routes to the latest location of a mobile sink with minimal network overhead. It partitions the sensor field into a virtual grid of $\mathrm{K}$ equal sized cells and constructs a virtual backbone network comprised of all the cell-headers. The nodes near to the centre of cell header will be responsible to collect the data and deliver to mobile sink using the backbone network. The goal behind such virtual structure construction is to minimize the routes re-adjustment cost due to sink mobility so that the observed data is delivered to the mobile sink in an energy efficient way.

The mobile sink moves randomly along the periphery of the sensor field in counter clockwise and communicates with the border cell-headers for data collection. The routes readjustment process is governed by a set of rules to dynamically cope with the sink mobility. For the route re- 
adjustment the biogeography based optimization approach is included with VGDRA for longer lifetime of network.

The rest of this paper is organized as follows: Details about the related work encompassing the various approaches in literature that deal with data delivery to a mobile sink in WSN presented in Section 2. Section 3 presents VGDRA scheme with the approach BBO in detail. To evaluate the performance of new VGDRA-BBO scheme, simulation setup and results are presented in Section 4. Finally, in Section 5 conclusion and future works of the research paper described.

\section{RELATED WORK}

Several virtual infrastructure based data dissemination protocols have been proposed for mobile sink based WSN in the last decade. Based on the mobility pattern exhibited by the sink in the sensor field, the data collection or dissemination schemes can be classified into controlled and uncontrolled sink mobility schemes. This paper considers the uncontrolled sink mobility environments and in the following lines, we briefly describe the related works in this context including their methodology and the relative strengths and weaknesses.

For mobile sink based wireless sensor network virtual infrastructure strategy are most efficient. Virtual infrastructure can achieve better scalability and energy efficiency [2]. Virtual infrastructure can be classified in to two types such as rendezvous based and backbone based. Backbone based approach is a kind of self organizing scheme but in rendezvous structure a localized rendezvous area defined within the sensor field [2]. In the present scenario lots of rendezvous or backbone based virtual infrastructure routing schemes are available. Virtual Grid Based Dynamic Routes Adjustment Scheme (VGDRA) and Virtual Circle Combined Straight Routing (VCCSR) are two data dissemination protocol used in a mobile sink based wireless sensor network.

Chen et al. [7] presented a converge-cast tree algorithm called Virtual Circle Combined Straight Routing (VCCSR) that constructs a virtual structure comprised of virtual circles and straight lines. A set of nodes are set as cluster heads along these virtual circles and straight lines. Together the set of cluster-heads form a virtual backbone network. The sink circulates the sensor field and maintains communication with the border cluster-heads for data collection. The clusterheads in VCCSR follow a set of communication rules to minimize the routes re-adjustment cost in propagating the sink's latest location information. VCCSR scheme although reduces the routes reconstruction cost in handling the sink mobility, however, the cluster-head at the centre of the sensor field being the focal point in routes re-adjustment process, depletes its energy much earlier.

Oh et al. suggested a scheme based on data dissemination known as Backbone-based Virtual Infrastructure (BVI) in [8], which makes use of single-level multi-hop clustering and points to reduce the total number of clusters.

It employs HEED [9] for clustering in which main concern is given to residual energy level of nodes for electing the $\mathrm{CH}$ nodes. The multi-hop clustering is a fine approach to reduce the number of clusters, on the other hand, the root node which is the focus in routes adjustments generates early energy depletion which reduces the lifetime of network.

The approach has been proposed in Line-Based Data Dissemination (LBDD) [10] that makes a vertical line by dividing the sensor field into two equal sized blocks.
Quadtree-based Data Dissemination (QBDD) scheme was suggested by Mir and Ko in [11], a node upon detecting an event calculates a set of rendezvous points (RPs) by successively partitioning the physical network space into four quadrants of uniform sizes. After partitioning the network, QDD routes the observed data to those nodes which are close to the centroid of each partition. The mobile sink disseminates the query packet using the same strategy by querying the node at closest RP first, followed by the subsequent RP nodes till it reaches the required data reportit also results in early energy depletion of nodes. This method also reduces the overall network lifetime.

A further approach called Virtual grid based Two-Tier Data Dissemination (TTDD) in [12] where forms a uniform per source node virtual grid structure covering the complete sensor field. TTDD prevents the flooding of the sink's topological updates but having the disadvantage of per source node virtual grid construction that reduces the network lifetime.

Abdul Waheed Khan, Abdul Hanan Abdullah Mohammad Abdur Razzaque, and Javed Iqbal Bangash [15] Proposed VGDRA Scheme of virtual infrastructure based data dissemination, that cause least communication cost and also maintains nearly optimal routes to the latest location of the mobile sink. The VGDRA scheme partitions the sensor field into a virtual grid and constructs a virtual backbone structure comprised of the cellheader nodes. A mobile sink when moves around the sensor field keep on changes its location and interacts with the nearest border-line cell-header for data collection. Using a set of communication rules, only a limited number of the cellheaders take part in route reconstruction process thereby reducing the overall communication cost.

Biogeography-Based Optimization (BBO) [16] was proposed by Simon, D. BBO is a new evolution algorithm developed for the global optimization. It is inspired by the immigration and emigration of species between islands (or habitats) in search of more compatible islands. In proposed work, the Biogeography-Based Optimization algorithm [17] is used for the dynamic deployment problem of WSNs. reason for using the optimization algorithm is to maximize the coverage rate of the network.

The mobile sink node comprises the subsequent data dissemination a challenging task due to limited resources containing sensor nodes. The infrequent dissemination results in poor data delivery performance lacking in using optimal routes. So, in this research work to optimize the trade-off between network's life time and data driven performance virtual grid based dissemination is proposed for event driven scenario.

\section{VGDRA-BBO SCHEME}

The VGDRA scheme constructs the virtual grid structure by first partitioning the sensor field into several uniform sized cells based on the number of nodes in the sensor field. The mobile sink node randomly moves in counter clockwise direction in periphery of sensor network.

A set of nodes close to the centre of the cells are appointed as cell-headers which keeps the track of latest location of mobile node and free the rest of nodes to take part in routereadjustment. Here given $N$ number of nodes, (see Figure 1) the VGDRA scheme partitions the sensor field into $K$ uniform sized cells as $\mathrm{K}=\left(4, \mathrm{~N}^{*} 0.05<=6\right)$. After the sensor network partitioning, now VGDRA scheme appoints a set of nodes as cell headers. When any event occurs in the field nodes need to 
setup their data delivery routes in accordance to latest location of the mobile sink. Adjacent cell-headers communicate with each other via gateway nodes. The set of cell-headers nodes together with the gateway nodes constructs the virtual backbone structure. The nodes which will participate in packet delivery will remain in active state and others will be sleep mode.

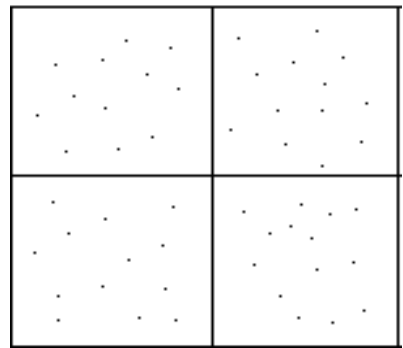

Fig.1 Shows network partitioning into various uniform sized cells for $N=\mathbf{5 0}$.

In existing VGDRA no specific AI or Genetic based routing scheme is implemented so we take the BBO Scheme to check the lifetime and throughput effect on existing system.

\subsection{Biogeography-Based Optimization}

As the name suggests, BBO is a population based global optimization technique developed on the basis of the science of biogeography i.e. study of the distribution of animals and plants among different habitats over time and space [17]. .the reason behind using $\mathrm{BBO}$ is that:

- $\quad \mathrm{BBO}$ has a way of sharing information between solutions.

- $\quad$ BBO solutions survive forever.

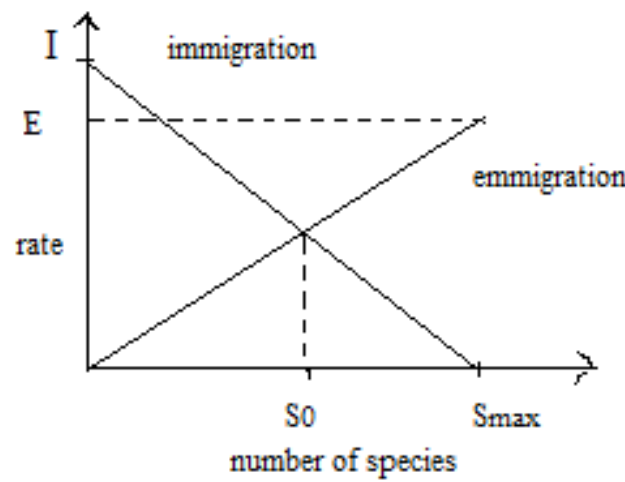

Fig.2 Linear migration relationship of an island. The equilibrium number of species is S0, where the immigration and emigration rates are identical.

Let's consider that there is a problem and a set of solutions which are represented in the form of vectors. Now suppose there is a way to assessing the accurate solutions. Accurate solutions are those having high value of ISI, and poor solutions are belongs to low ISI. Note that ISI is the same as "fitness" in other population based optimization algorithms. In biogeography, species migrate between islands. However, in $\mathrm{BBO}[20]$ solution features (SIVs) migrates between islands. The migration probabilities are based on a curve similar to that shown in Figure 2, but considering simplicity assume that all solutions (islands) have similar migration curves with $\mathrm{E}=\mathrm{I}$. In this approach, for each feature in each solution, we use the immigration curve to probabilistically decide whether or not to immigrate. If immigration is selected for a given solution feature, then the emigrating island is selected probabilistically. The notation $y_{k}(s)$ is used to denote the $s_{t h}$ feature of the kth population member represented as in figure 3. Migration and mutation of the entire population take place before any of the solutions are replaced in the population, which requires the use of the temporary population vector $\mathrm{w}$ as shown in BBO Algorithm.

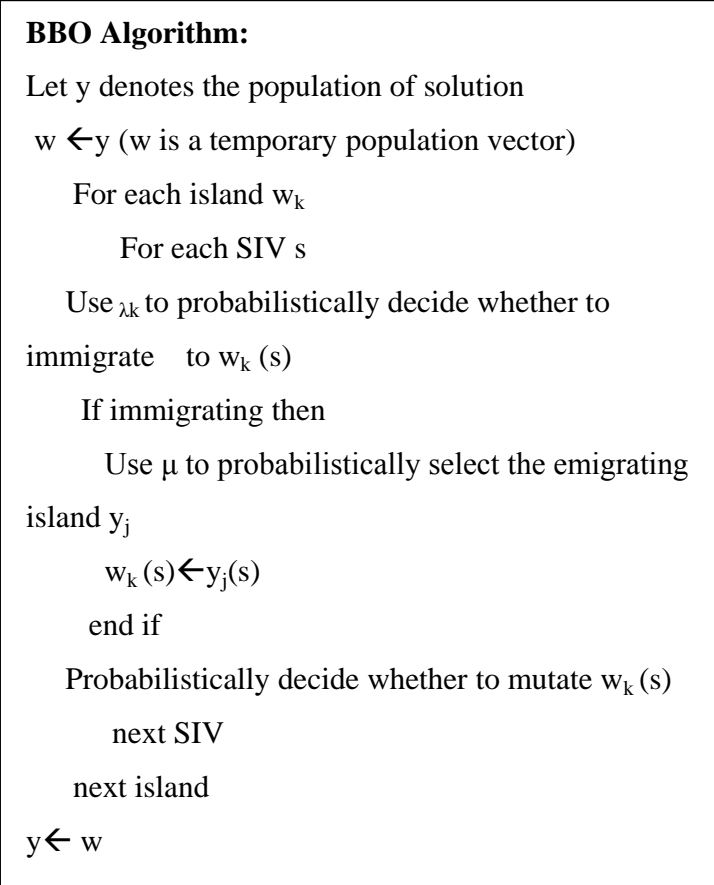

Fig.3 The above algorithm is one generation of the partial immigration-based $\mathrm{BBO}$ algorithm. $\mathrm{Y}$ is the entire population of solutions, $y_{k}$ is the $k_{t h}$ solution, and $y_{k}(s)$ is the $s_{t h}$ feature of $y_{k}$.

\subsection{Proposed Methodology}

Methodology used in VGDRA-BBO for energy enhancement is described in the following steps:

Step1: Define the area where the sensor network to be created.

Step 2: Initializing WSN parameter.

Step 3: Deploying No. of Nodes in the network.

Step 4: Implementing existing VGDRA Protocol.

Step 5: Initialize BBO parameter for routing.

Step 6: Defining Migration and Emigration rate.

Step 7: Routing update with each round.

Step 8: Checking Lifetime and Data Consumption.

Step 9: Comparison with previous VGDRA Scheme.

The various parameters in support of WSN network are taken are described in following (see Table 1):

Table 1 System Parameters Value

\begin{tabular}{|c|c|c|}
\hline Sr. No. & Parameter & Value \\
\hline 1 & No.of nodes & 50 \\
\hline 2 & Environment Size & $1300 * 1000$ \\
\hline
\end{tabular}




\begin{tabular}{|c|c|c|}
\hline 3 & Packet Queue Size & 300 \\
\hline 4 & $E_{\text {elect }}$ & $50 \mathrm{~nJ}$ \\
\hline 5 & No.of Cell Heads & 4 \\
\hline
\end{tabular}

\section{SIMULATION AND RESULTS}

Here, the simulation results are shown using NS-2, taken 50 no. of nodes for the visualisation. The nodes are randomly deployed in a sensor field area of $1300 \times 1000$ dimension. A mobile sink moves around the sensor field counter clockwise and periodically broadcasts the packets. This paper uses the ns2 simulator to evaluate the existing work of VGDRA compared with VGDRA-BBO.

\subsection{Simulation result for Network Lifetime}

The network lifetime is defined as the time elapsed since the nodes deployment till the first node dies due to energy depletion. From experiments, it is estimated that the network lifetime of proposed scheme is better than existing VGDRA scheme in terms of the number of rounds of the mobile sink around the sensor field till the first node in the network dies due to energy depletion. In Figure 4, the proposed VGDRABBO scheme lifetime and existed VGDRA scheme comparisons are presented as dead nodes to lifetime in hours at different network sizes.

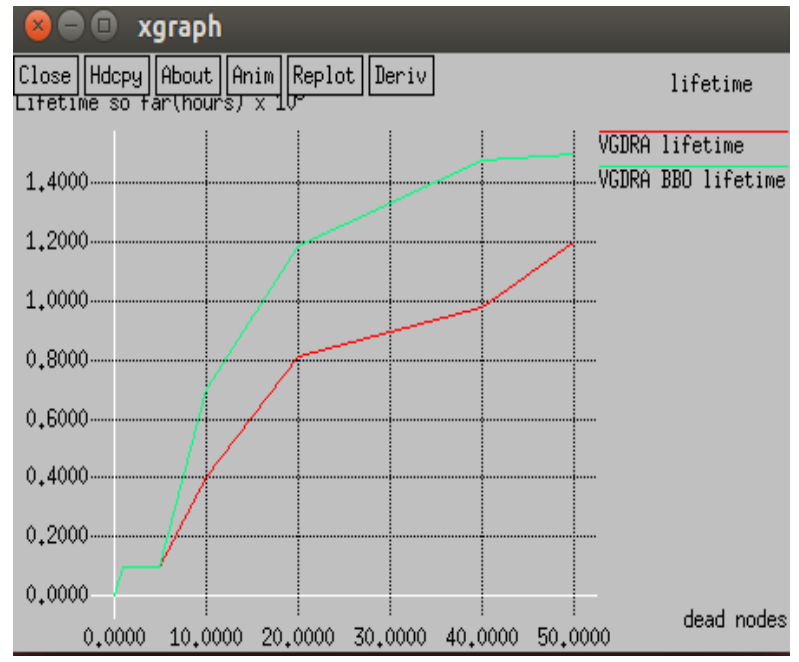

Fig 4: Proposed VGDRA-BBO scheme Lifetime comparison with existing VGDRA

\subsection{Simulation Result for Throughput: The} network throughput is the rate of successful packet delivery over the communication channel. Throughput is measured in bits per second. As presented the comparison between existed VGDRA scheme and proposed VGDRA-BBO scheme in Figure 5 in terms of different iterations to the no. of packet transmission per unit of time.

\section{Throughput=(Size of the packet/Transmission time $)$}

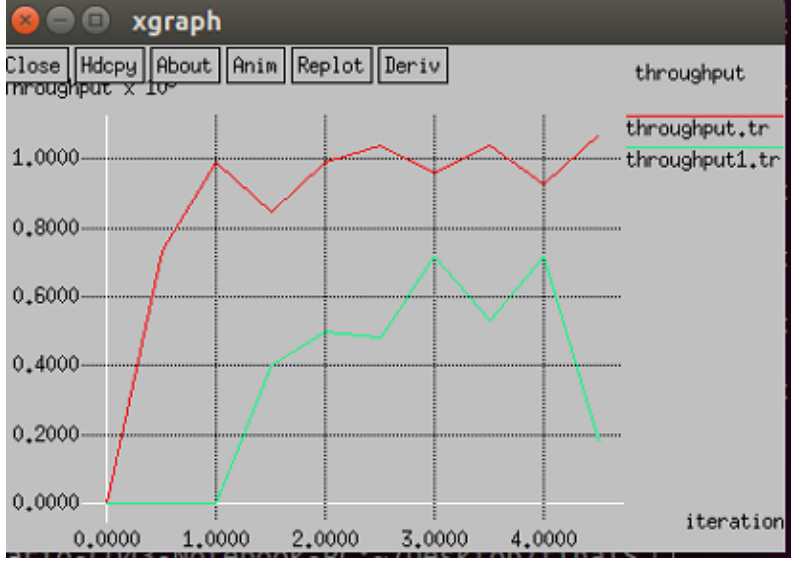

Fig 5: The VGDRA throughput in comparison with proposed VGDRA-BBO scheme

The comparison between the results of existing VGDRA and proposed work on the existing scheme results are elaborated further in the Table 3 .

Table 3 Comparison between Proposed and Existing Schemes

\begin{tabular}{|c|c|c|}
\hline Parameters & Proposed Scheme & $\begin{array}{c}\text { Existing } \\
\text { Scheme(VGDRA) }\end{array}$ \\
\hline No.of Nodes & 50 & 50 \\
\hline Lifetime & $1.5^{*} 10^{4}$ hours & $1.2^{*} 10^{4}$ hours \\
\hline Throughput & Less & More \\
\hline Efficiency & More & Less \\
\hline
\end{tabular}

As the less number of nodes participates in route optimization of our proposed scheme, the less time required for transmission in iterations as shown in throughput comparison see Figure 5. Due to this reason the nodes which yet participated in routing will be considered in next iteration for transmission. This is how the efficiency of the proposed network can be considered more.

\section{CONCLUSION}

In this paper, work is considered on the improvement in life time of Virtual Grid based Dynamic Routes Adjustment (VGDRA) scheme maintaining nearly optimal routes to the latest location of the mobile sink. In terms of nodes energy consumption, the simulation results show the various factor improving existing VGDRA scheme for the sensor field. In this work the routing is taken into account by adapting BBO strategy to create grid and data dissemination balancing the energy consumption in response to longer lifetime of WSN.

In Future work, multiple mobile sinks can be considered with different speeds to check efficiency and quality of service of the network by using the proposed scheme.

\section{REFERENCES}

[1] R. C. Shah, S. Roy, S. Jain, and W. Brunette, "Data MULEs: Modeling and analysis of a three-tier architecture for sparse sensor networks," in Ad Hoc Netw., vol. 1. 2003, pp. 215-233. 
[2] S. R. Gandham, M. Dawande, R. Prakash, and S. Venkatesan, "Energy efficient schemes for wireless sensor networks with multiple mobile base stations," in Proc. IEEE Global Telecommun. Conf. (GLOBECOM), vol. 1. Dec. 2003, pp. 377-381.

[3] W. Khan, A. H. Abdullah, M. H. Anisi, and J. I. Bangash, "A comprehensive study of data collection schemes using mobile sinks in wireless sensor networks," Sensors, vol. 14, no. 2, pp. 2510-2548, 2014.

[4] M. Di Francesco, S. K. Das, and G. Anastasi, "Data collection in wireless sensor networks with mobile elements," ACM Trans. Sensor Netw., vol. 8, no. 1, pp. 1-31, Aug. 2011.

[5] Chalermek, R. Govindan, and D. Estrin, "Directed diffusion: A scalable and robust communication paradigm for sensor networks," in Proc. ACM SIGMOBILE Int. Conf. Mobile Comput. Netw. (MOBICOM), 2000, pp. 56-67.

[6] E. B. Hamida and G. Chelius, "Strategies for data dissemination to mobile sinks in wireless sensor networks," IEEE Wireless Commun., vol. 15, no. 6, pp. 31-37, Dec. 2008.

[7] T.-S. Chen, H.-W. Tsai, Y.-H. Chang, and T.-C. Chen, "Geographic convergecast using mobile sink in wireless sensor networks," Comput. Commun., vol. 36, no. 4, pp. 445-458, Feb. 2013.

[8] S. Oh, E. Lee, S. Park, J. Jung, and S.-H. Kim, "Communication scheme to support sink mobility in multi-hop clustered wireless sensor networks," in Proc. 24th IEEE Int. Conf. Adv. Inf. Netw. Appl., Apr. 2010, pp. 866-872.

[9] O. Younis and S. Fahmy, "HEED: A hybrid, energyefficient, distributed clustering approach for ad hoc sensor networks," IEEE Trans. Mobile Comput., vol. 3, no. 4, pp. 366-379, Oct. 2004.
[10] B. Hamida and G. Chelius, "A line-based data dissemination protocol for wireless sensor networks with mobile sink," in Proc. IEEE Int. Conf. Commun., May 2008, pp. 2201-2205.

[11] Z. H. Mir and Y.-B. Ko, "A quadtree-based data dissemination protocol for wireless sensor networks with mobile sinks," in Proc. Personal Wireless Commun., 2006, pp. 447-458.

[12] H. Luo, F. Ye, J. Cheng, S. Lu, and L. Zhang, "TTDD: Two-tier data dissemination in large-scale wireless sensor networks," Wireless Netw., vol. 11, nos. 1-2, pp. 161-175, Jan. 2005.

[13] W. B. Heinzelman, A. P. Chandrakasan, S. Member, and H. Balakrishnan, "An application-specific protocol architecture for wireless microsensor networks," IEEE Trans. Wireless Commun., vol. 1, no. 4, pp. 660-670, Oct. 2002

[14] Manjeshwar and D. P. Agrawal, "TEEN: A routing protocol for enhanced efficiency in wireless sensor networks," in Proc. 15 ${ }^{\text {th }}$ Int. Parallel Distrib. Process. Symp. (IPDPS), vol. 1. Apr. 2000, pp. 2009-2015.

[15] Abdul Waheed Khan, Abdul Hanan Abdullah Mohammad Abdur Razzaque, and Javed Iqbal Bangash "VGDRA: A Virtual Grid-Based Dynamic Routes Adjustment Scheme for Mobile Sink-Based Wireless Sensor Networks" IEEE sensors journal, vol. 15, no. 1, january 2015

[16] Simon, D, Biogeography based Optimization IEEE Trans. Evol. Comput. 2008, 6, 702-713.

[17] Bipandeep Singh, “An Improved Energy-Efficient BBOBased PEGASIS Protocol in Wireless Sensors Network" in nt. Journal of Engineering Research and Applications, Vol. 4, Issue 3( Version 1), March 2014, pp.470-474 